\title{
Wind Turbine Fault Diagnosis System Based on A Fuzzy Expert System
}

\author{
Wenguang Yang, Dongxiang Jiang
}

State Key Laboratory of Control and Simulation of Power System and Generation Equipments, Department of Thermal Engineering, Tsinghua University, Beijing, 100084, China

ywg10@mails.tsinghua.edu.cn, jiangdx@tsinghua.edu.cn

Keywords: Wind turbine, Fault diagnosis system, Fuzzy expert system, Fuzzy Rete algorithm

\begin{abstract}
A fuzzy expert system which can simultaneously support many kinds of rules including fuzzy production rule, fault tree and decision tree in a unified framework are researched. Fuzzy rete algorithm is adopted to speed up the fuzzy reasoning chain. A wind turbine diagnosis expert system is developed based on the fuzzy expert system. Experiment data is employed to verify the feasibility of the inference engine, and the results show that with proper knowledge imported the misdiagnosis rate is low, the inference process can be exported and the match degree of the health rule can be used as a health state indicator.
\end{abstract}

\section{Introduction}

The world-wide wind power installed capacity has exceeded 360GW by the end of 2014 [1]. Wind turbines are often located in remote areas and their working condition is poor which make them suffer high failure rate and hard to repair. All these factors lead the cost of operation and maintenance $(\mathrm{OM})$ of wind turbine is quite high, which is estimated to be $10-15 \%$ of the total income for onshore wind turbines, and $20-25 \%$ for offshore wind turbines [2]. Wind turbine fault diagnosis, as one of the advanced OM technologies show great benefit for such situations [3].

The development of fault diagnosis system is one of the research areas of fault diagnosis technology. Lots of representative works can be found in this research area. Zaher, A. S. et al. described the use of a combination of anomaly detection and data-trending techniques encapsulated in a multi-agent framework for the development of a fault detection system for wind turbines [4]. Zhou, J. H. et al. proposed a hierarchical rule-based fault detection system which comprises of a knowledge base coupled with an inference engine [5]. Chen, C. Z. et al. constructed a large-scale wind turbine fault diagnostic expert system which was constructed based on expert system tool CLIPS [6]. Garcia etc. presented the architecture of a new predictive maintenance system, called SIMAP, based on artificial intelligent techniques [7]. However, intelligent methods in these works have some drawbacks. The inference process of ANN is opacity and hard to explain. Fuzzy system does not support reasoning chain. Also, CLIPS does not support fuzzy reasoning [8].

Compared with the other methods, the inference engine and knowledge base of expert system are separated, so using expert system as the top-level controller for diagnosis process is very attractive. We can integrate the other analysis methods into the system by defining the outputs of these methods as features in the knowledge base, and the expert experience can also be imported into the knowledge base. In this paper, we research and develop a fuzzy expert system, which can simultaneously use many types of rules such as fuzzy production rules, decision tree and fault tree, and build an advanced wind turbine fault diagnosis system based on this fuzzy expert system.

\section{A Fuzzy Expert System}

Fig. 1 shows the structure of the designed fuzzy expert system. Fuzzy symbol manager, rule base and the fuzzy inference engine set (FIES) make this expert system different from the traditional production systems. 


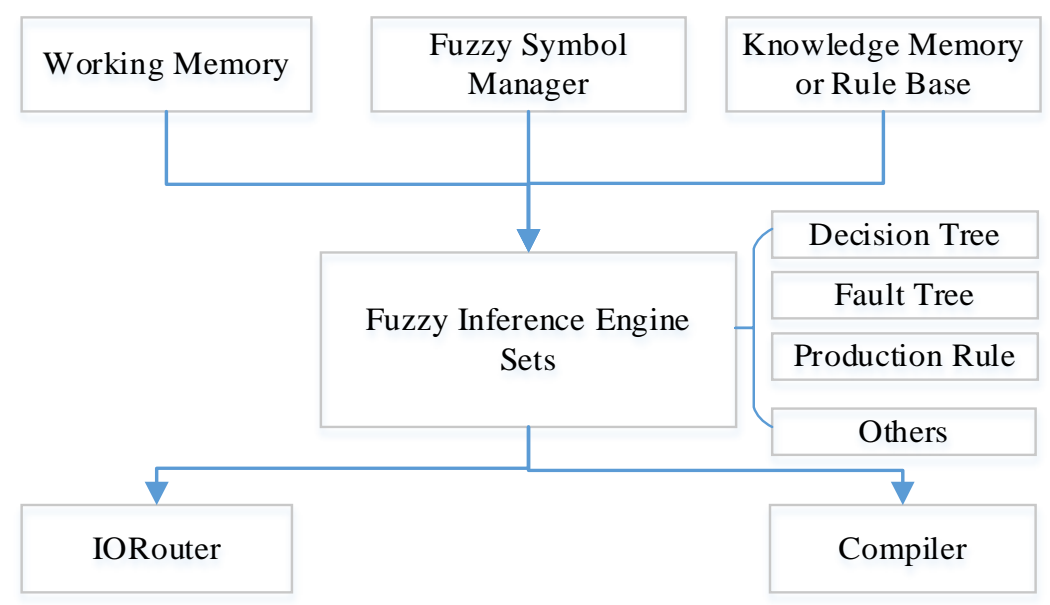

Fig. 1 Architecture of the fuzzy expert system

Fuzzy symbol manager stores all the fuzzy terms which are used by facts and rules, and provides the function of various fuzzy operations used by inference engines. Rule base is a kind of extension to traditional production memory, which can store not only production rules but also logic rules and forward trees etc. Fuzzy inference engine set consists of many parallel inference engines, all the inference engines share the same working memory and rule base, and each inference engine can make use of a special rule representation. Now we already have three build-in inference engines, a forward tree based inference engine, a fault tree based one and a fuzzy production rule based one. A modern script language is designed for the expert system to make it easy to use, and a compiler is developed for its recognition.

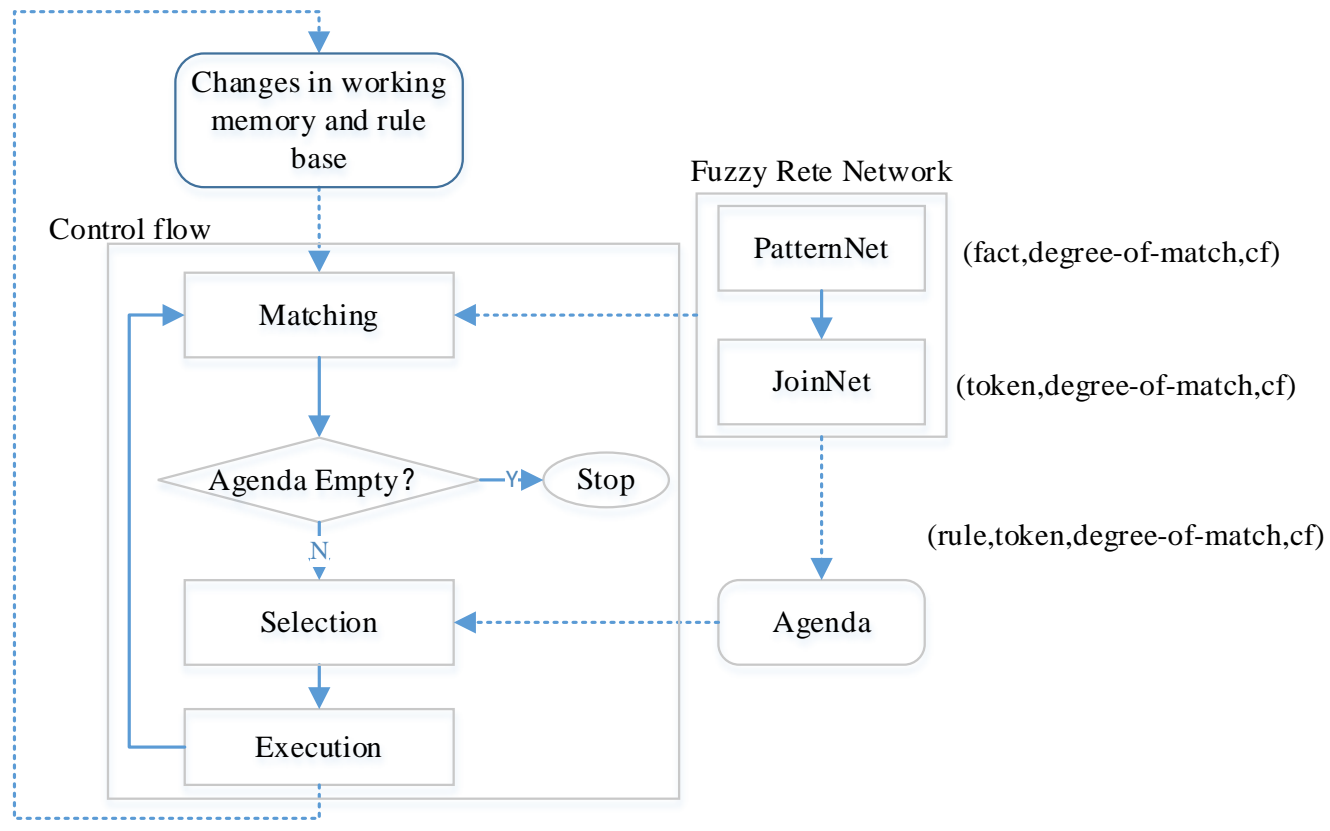

Fig. 2 Inference process based on fuzzy rete algorithm

The innovations of this architecture include, users can take advantages of hybrid knowledge representation by splitting the whole inference process into several sections and controlling the active sequence among these engines, and can develop their own knowledge representation and inference engine for their special requirements with little effort.

Fig. 2 depicts the overall control flow and the structure of our inference engines. Each cycle of the inference engine consists of three steps, called matching, selection, and execution respectively for short. The key technology of inference engine lays in its match process. Rete algorithm is a fast algorithm for the pattern match problem between many patterns and many objects [9]. We modified the fuzzy Rete network proposed by Juiyao Pan [10] for our match process. 


\section{A Wind Turbine Fault Diagnosis System}

Our wind turbine fault diagnosis system contains two parts, the diagnosis server and the client software (see Fig. 3). The server is responsible for data collection, data processing including condition monitoring and fault diagnosis, management of many kinds of data and knowledge management. Client software is used for users to get the wind turbine monitoring and diagnosis services through network. The services (see Fig. 4) provided by client software include: real-time monitoring of the performance status of wind turbine; trigger alarm when anomaly detected; help to diagnose fault and take measures quickly; query the historical data to mine knowledge and help arrange maintenance; and manage the knowledge in knowledge base.

Fig. 3 shows the architecture of the server, which mainly consists of five parts, data collector, real-time database management system, knowledge base, result database and the inference engine.

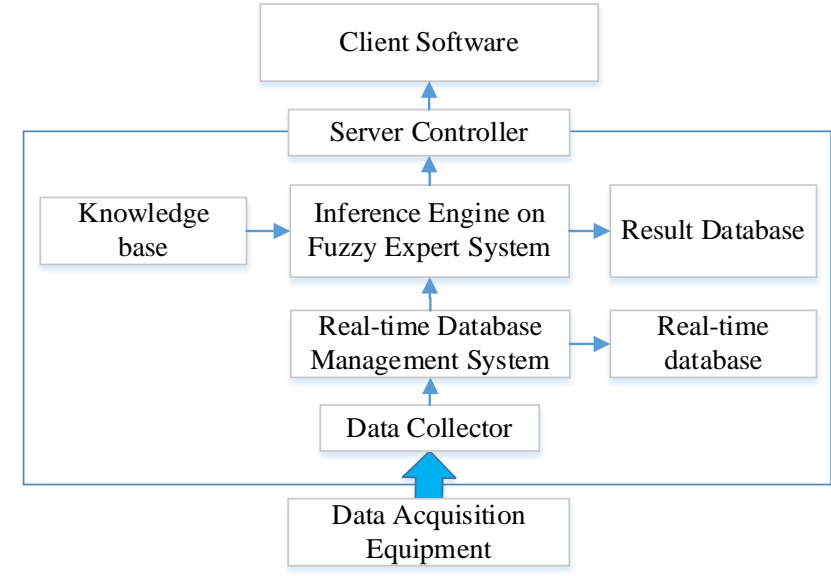

Fig. 3 Architecture of the server

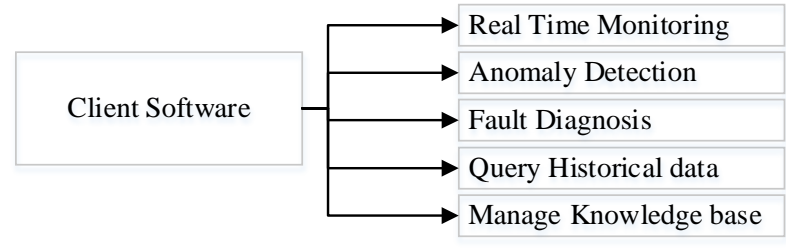

Fig. 4 Functions provided by client software

Data collector can get raw data from SCADA or any other third-party systems, and extract features by signal analysis. Real-time database manage raw data and features extracted. Knowledge base stores a lot of ontologies and its complex relationships, among them structure information, features, thresholds and rules are the most important ones. Registering features in the knowledge base makes the system very extensible to add new sensors and new data processing techniques. Result database stores the processing results of the inference engine, mainly includes the detailed information of anomalies detected and the faults diagnosed. These information can be used for life prediction and maintenance arrangement. Inference engine is built based on the developed fuzzy expert system. It reads knowledge from knowledge base, changes its format, feeds the knowledge into the fuzzy expert system, starts the reasoning process and explains the outputs.

\section{System Verification by Experiment}

Experiment and Knowledge Acquisition. Knowledge and data are needed for the system verification. In this study, the data from a wind turbine fault simulation experiment is used. The details about the experiment can be found in [11]. In our system verification process, only three kinds of fault with wind speed around $8 \mathrm{~m} / \mathrm{s}$ are used, and three features are adopted, which are rotor rotate speed, generator electrical power, and shaft vertical displacement vibration peak to peak value. These features are represented by RotateSpeed, Power and Vpph for short. Table 1 lists parts of the data samples chosen from [11]. Fig. 5 shows the scatter plot of the experiment data.

Table 1 Experiment data samples

\begin{tabular}{l|c|c|c|c|c}
\hline State & Identifier & $\begin{array}{c}\text { Wind } \\
\text { speed[m/s] }\end{array}$ & $\begin{array}{c}\text { RotateSpeed } \\
{[\mathrm{rpm}]}\end{array}$ & Power $[\mathrm{W}]$ & $\begin{array}{c}\text { Vpph } \\
{[\mu \mathrm{m}]}\end{array}$ \\
\hline Normal & Normal & 8.1 & 267 & 25.4 & 169 \\
\hline Mass unbalance & Unbalance & 8.4 & 258 & 18 & 162 \\
\hline Airfoil changed & Airfoil & 8.4 & 250 & 10.8 & 179 \\
\hline
\end{tabular}




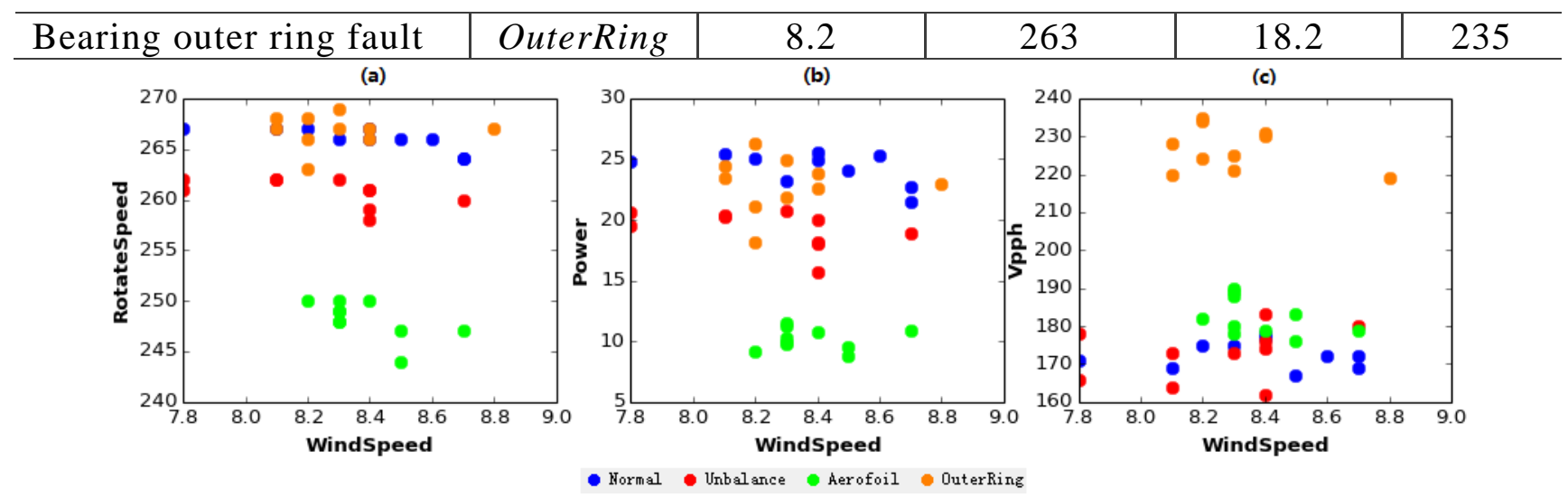

Fig. 5 Scatter plot of the experiment data

For each feature, one lower limit and one upper limit for each feature are learned and three fuzzy thresholds, represented by normal, low and high, are built based on the above two thresholds. Suppose the two thresholds lower limit and upper limit, represented by $l$ and $u$ respectively, and $a, b$, $c, d$ are parameters. Then the membership functions for the three fuzzy sets can be defined as follow,

$$
\begin{aligned}
& \text { low }=\{1 / l-a, 0.5 / l, 0 / l+b\} \\
& \text { normal }=\{0 / l-a, 0.5 / l, 1 / l+b, 0 / u-c, 0.5 / u, 1 / u+d\} \\
& \text { high }=\{0 / u-c, 0.5 / u, 1 / u+d\} .
\end{aligned}
$$

Table 2 Alarm thresholds and parameters involved in fuzzy thresholds for each feature

\begin{tabular}{l|c|c|c|c|c|c|c}
\hline Feature & Lower limit & Upper limit & $\mathrm{a}$ & $\mathrm{b}$ & $\mathrm{c}$ & $\mathrm{d}$ \\
\hline RotateSpeed & 255 & 270 & 1 & 1 & 1 & 1 \\
\hline Power & 21 & 26 & 0.1 & 0.1 & 0.1 & 0.1 \\
\hline Vpph & 160 & 200 & 1 & 1 & 1 & 1 \\
\hline
\end{tabular}

Fig. 6 Membership functions of each feature

For our experiment data, the thresholds and the parameters involved in fuzzy thresholds are listed in Table 2. The membership functions are shown in Fig. 6.

Fuzzy rules for faults extracted by cluster analysis are given as follow,

Rule 1: If Power is normal, RotateSpeed is normal and Vpph is normal, then system is healthy;

Rule 2: If Power is lower, RotateSpeed is normal and Vpph is normal, then fault is unbalance;

Rule 3: If Power is lower, RotateSpeed is lower and Vpph is high, then fault is Bearing;

Rule 4: If Power is normal, RotateSpeed is normal and Vpph is high, then fault is Airfoil.

As we mentioned before, our fuzzy expert system can take advantage of several kinds of rules, therefore, a decision tree is extracted to verify this function, which is shown in Fig. 7. 


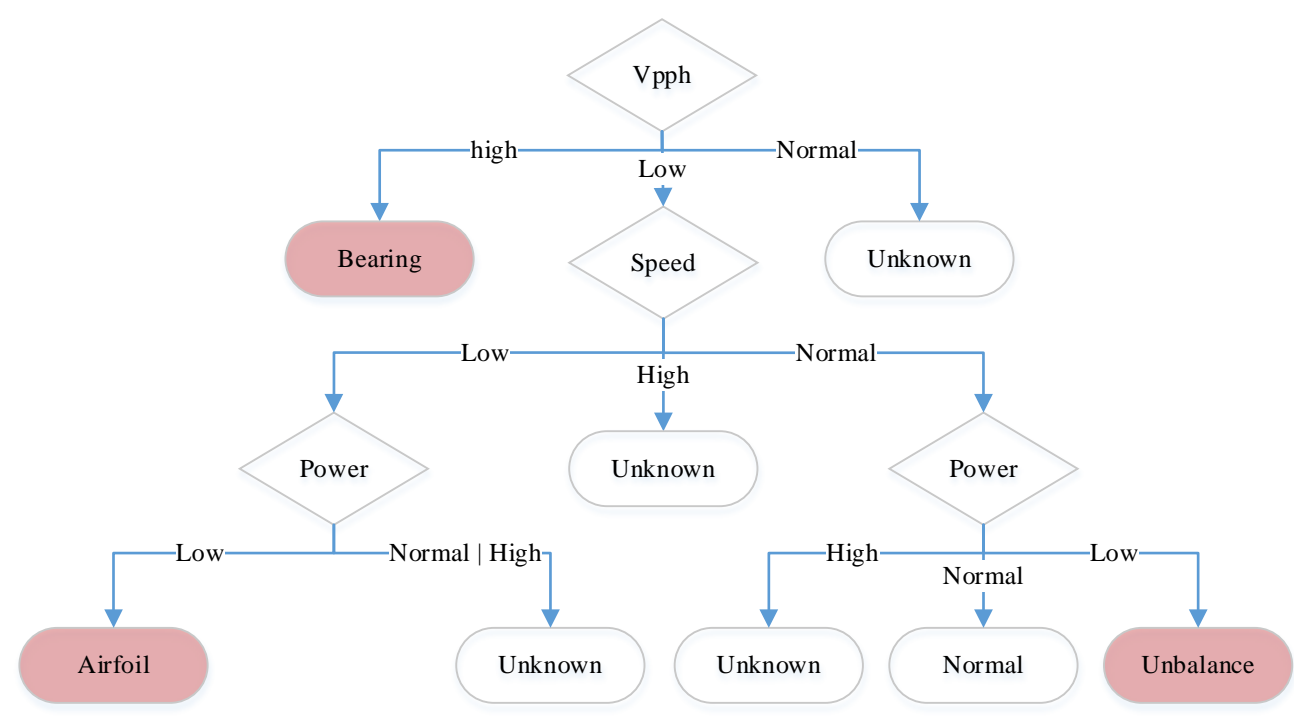

Fig. 7 Decision tree extracted from the experiment data

Results and Discussion. The features, faults, fuzzy thresholds, fuzzy rules and decision tree are imported into the knowledge base through the knowledge base management function provided by another software tool. Users don't need to learn the grammar of the script of the fuzzy expert system, since the inference engine can generate it from the knowledge base automatically in runtime. For example, the auto-generated fuzzy rule for unbalance fault is shown as below,

\section{[module Imain]}

\section{lrule rule1\{}

ce: Power = lower; ce: RotateSpeed = normal; ce: Vpph = normal;

\}$=>\{$

Fault\{name = "unbalance" with_match\};

\} .

We develop a virtual wind turbine to generate real-time fault simulation data, and modify the collector in the server to read data from it. Client software is employed to obtain the analysis results.

Test results show that the misdiagnosis rate is zero for all the experiment data, and the match degree and inference process can be exported. If we randomly generate a normal sample which near to the thresholds, we could found that the degree of match change between 0 and 1 , which means that, this output parameter can be used as a health state indicator. 1 means total health, 0 means failure, a number between 0 and 1 means sub-health. The changes of degree of match can also be found in fault random simulation. If we randomly generate an abnormal sample which does not match any rules in our knowledge base, the result says unknown fault is given. Thus the feasibility of the fuzzy expert system and the functions of the wind turbine diagnosis system was verified.

\section{Summary}

Wind turbine fault diagnosis is a promising research area. In this paper we research and develop a fuzzy expert system and a wind turbine fault diagnosis system. The fuzzy expert system supports many kinds of rules including fuzzy production rule, fault tree and decision tree in a unified framework, fuzzy rete algorithm is adopted to speed up the fuzzy reasoning chain and users can easily develop their own knowledge representation and inference engine. The wind turbine diagnosis system use the fuzzy expert system in its inference engine which makes it intelligent and flexible. Experiment data from a wind turbine fault simulation test rig is used to test the designed system. The test results show that, with proper knowledge imported, the misdiagnosis rate for test samples is low, which verifies the feasibility of the expert system we developed.

For the following work, we will obtain the knowledge for real wind turbines by many approaches including carrying out quantitative research of the wind turbine fault mechanism. 


\section{Acknowledgements}

This work was supported by the National Natural Science Foundation of China (Grant 51174273).

\section{References}

[1] W. W. E. Association, "WWEA half year report 2014," (2014).

[2] L. Bin, L. Yaoyu, W. Xin, and Z. Yang, "A review of recent advances in wind turbine condition monitoring and fault diagnosis," in Power Electronics and Machines in Wind Applications, 2009. PEMWA 2009. IEEE, Lincoln, NE, (2009), pp. 1-7.

[3] P. Takoutsing, R. Wamkeue, M. Ouhrouche, F. Slaoui-Hasnaoui, T. Tameghe, and G. Ekemb, "Wind Turbine Condition Monitoring: State-of-the-Art Review, New Trends, and Future Challenges," Energies, vol. 7, pp. 2595-2630, (2014-04-22) (2014).

[4] A. S. Zaher, A. S. Zaher, S. D. J. McArthur, and S. D. J. McArthur, "A Multi-Agent Fault Detection System for Wind Turbine Defect Recognition and Diagnosis,": IEEE, (2007), pp. 22 - 27.

[5] J. H. Zhou, L. Wee and Z. W. Zhong, "A knowledge base system for rotary equipment fault detection and diagnosis," in Control Automation Robotics \& Vision (ICARCV), 2010 11th International Conference on, Singapore, (2010), pp. 1335-1340.

[6] C. Chen and Y. Li, "The fault diagnosis of large-scale wind turbine based on expert system,", (2011), p. 82852M-82852M.

[7] M. C. Garcia, M. A. Sanz-Bobi and J. Del Pico, "SIMAP: Intelligent System for Predictive Maintenance Application to the health condition monitoring of a windturbine gearbox," Computers in Industry, vol. 57, pp. 552-568, (2006).

[8] G. Riley, "CLIPS Architecture Manual," Software Technology Branch, Lyndon B Johnson Space (1992).

[9] C. L. FORGY, "Rete: A fast algorithm for the many pattern many object pattern match problem," ARTIFICIAL INTELLIGENCE, vol. 19, pp. 17-37, (1982).

[10]G. N. D. A. Juiyao Pan, "FuzzyShell: A large-scale expert system shell using fuzzy logic for uncertainty reasoning," IEEE TRANSACTIONS ON FUZZY SYSTEMS, vol. 6, pp. 563-581, (1998).

[11]D. J. Wenguang Yang, "Experimental Investigation and Analysis of Some Common Faults for Wind Turbine," in Machinery Failure Prevention Technology (MFPT) 2014 Virginia Beach, VA: The Society for Machinery Failure Prevention Technology (MFPT), (2014). 